\title{
SCHATTEN CLASSES OF MATRICES IN A GENERALIZED $\mathcal{B}\left(l_{2}\right)$
}

\author{
JitTi Rakbud and Pachara Chaisuriya
}

\begin{abstract}
In this paper, we study a generalization of the Banach space $\mathcal{B}\left(l_{2}\right)$ of all bounded linear operators on $l_{2}$. Over this space, we present some reasonable ways to define Schatten-type classes which are generalizations of the classical Schatten classes of compact operators on $l_{2}$.
\end{abstract}

\section{Introduction and preliminary results}

For a separable Hilbert space $\mathcal{H}$ and $1 \leq p \leq \infty$, the Schatten $p$-class, $\mathcal{C}_{p}$, is the class of all compact operators $\mathrm{A}$ on $\mathcal{H}$ such that the sequence $\left\{s_{n}(A)\right\}_{n=1}^{\infty}$ of singular values of $A$ belongs to $l_{p}$. Equipped with the norms $\|A\|_{p}=\left\|\left\{s_{n}(A)\right\}_{n=1}^{\infty}\right\|_{p}$, the classes $\mathcal{C}_{p}$ are Banach spaces. These were introduced, in [8], by von Neumann and Schatten as a completion of the tensor product $\mathcal{H} \otimes \mathcal{H}$ in various norms. Since then many mathematicians have contributed and extended their results, see [3, 4, 5] for references. In this paper, we give some reasonable ways to define Schatten-type classes which generalize the classical Schatten classes of compact operators on $l_{2}$.

Let $X$ be a compact Hausdorff space, and let $C(X)$ be the $C^{*}$-algebra of continuous complex-valued functions on $X$. In this paper, we denote the norm on $C(X)$ by $\|\cdot\|_{C(X)}$. In [6], Leo Livshits, Sing-Cheong Ong, and Sheng-Wang Wang defined the sequence spaces $l_{2}(C(X))$ and $l_{2}^{b}(C(X))$ as follows:

$l_{2}(C(X))=\left\{\left\{f_{k}\right\}_{k=1}^{\infty}: f_{k} \in C(X) \forall k,\left\{\sum_{k=1}^{n}\left|f_{k}\right|^{2}\right\}_{n=1}^{\infty}\right.$ converges in $\left.C(X)\right\}$,
$l_{2}^{b}(C(X))=\left\{\left\{f_{k}\right\}_{k=1}^{\infty}: f_{k} \in C(X) \forall k,\left\{\sum_{k=1}^{n}\left|f_{k}\right|^{2}\right\}_{n=1}^{\infty}\right.$ is bounded in $\left.C(X)\right\}$.

Received December 13, 2007.

2000 Mathematics Subject Classification. Primary 47L10; Secondary 47L20.

Key words and phrases. compact operator, Schatten $p$-class, singular values. 
In [7], J. Rakbud and P. Chaisuriya extended to $1 \leq p<\infty$ :

$l_{p}(C(X))=\left\{\left\{f_{k}\right\}_{k=1}^{\infty}: f_{k} \in C(X) \forall k,\left\{\sum_{k=1}^{n}\left|f_{k}\right|^{p}\right\}_{n=1}^{\infty}\right.$ converges in $\left.C(X)\right\}$,
$l_{p}^{b}(C(X))=\left\{\left\{f_{k}\right\}_{k=1}^{\infty}: f_{k} \in C(X) \forall k,\left\{\sum_{k=1}^{n}\left|f_{k}\right|^{p}\right\}_{n=1}^{\infty}\right.$ is bounded in $\left.C(X)\right\}$ and proved that $l_{p}(C(X))$ and $l_{p}^{b}(C(X))$ endowed with the norm

$$
\left\|\left\{f_{k}\right\}_{k=1}^{\infty}\right\|_{p}:=\sup _{t \in X}\left(\sum_{k=1}^{\infty}\left|f_{k}(t)\right|^{p}\right)^{\frac{1}{p}}
$$

are Banach spaces.

It is clear that $l_{p}(C(X)) \subseteq l_{p}^{b}(C(X))$. For the case where $X$ is finite, we have $l_{p}(C(X))=l_{p}^{b}(C(X))$. If $X$ is a singleton, then $l_{p}(C(X))=l_{p}^{b}(C(X))=l_{p}$. The following example shows that the inclusion $l_{p}(C(X)) \subseteq l_{p}^{b}(C(X))$ can be proper.

Example $1.1([6,7]) \cdot l_{p}(C(X)) \varsubsetneqq l_{p}^{b}(C(X))$. Let $X=[0,1]$ and for each $k \in \mathbb{N}$, let $f_{k}(t)=\left(t^{k}-t^{k+1}\right)^{\frac{1}{p}}$ for all $t \in[0,1]$. Let $f\langle p\rangle=\left\{f_{k}\right\}_{k=1}^{\infty}$. Then $f\langle p\rangle$ belongs to $l_{p}^{b}(C([0,1]))$, but does not belong to $l_{p}(C([0,1]))$.

Proposition $1.2([7])$. Let $f=\left\{f_{k}\right\}_{k=1}^{\infty}$ be a sequence over $C(X)$ with $f[t]:=$ $\left\{f_{k}(t)\right\}_{k=1}^{\infty} \in l_{p}$ for all $t \in X$. Then the following are equivalent.

(1) $f \in l_{p}(C(X))$.

(2) The function $t \mapsto f[t]$ from $X$ into $l_{p}$ is continuous.

(3) The function $t \mapsto\|f[t]\|_{p}$ from $X$ into $[0, \infty)$ is continuous.

Theorem $1.3([6,7])$. Let $g=\left\{g_{k}\right\}_{k=1}^{\infty}$ be a sequence in $C(X)$. Then

(1) $\left\{f_{k} g_{k}\right\}_{k=1}^{\infty} \in l_{1}(C(X))$ for all $f=\left\{f_{k}\right\}_{k=1}^{\infty} \in l_{2}(C(X))$ if and only if $g \in l_{2}^{b}(C(X))$. If $g \in l_{2}^{b}(C(X))$, then $\|g\|_{2}=\sup \left\{\left\|\left\{g_{k} f_{k}\right\}_{k=1}^{\infty}\right\|_{1}: f=\right.$ $\left.\left\{f_{k}\right\}_{k=1}^{\infty} \in l_{2}(C(X)),\|f\|_{2} \leq 1\right\}$.

(2) $\left\{f_{k} g_{k}\right\}_{k=1}^{\infty} \in l_{1}(C(X))$ for all $f=\left\{f_{k}\right\}_{k=1}^{\infty} \in l_{2}^{b}(C(X))$ if and only if $g \in l_{2}(C(X))$. If $g \in l_{2}(C(X))$, then $\|g\|_{2}=\sup \left\{\left\|\left\{g_{k} f_{k}\right\}_{k=1}^{\infty}\right\|_{1}: f=\right.$ $\left.\left\{f_{k}\right\}_{k=1}^{\infty} \in l_{2}^{b}(C(X)),\|f\|_{2} \leq 1\right\}$.

\section{A generalization of $\mathcal{B}\left(l_{2}\right)$}

We say that a matrix $A=\left[a_{j k}\right]$ with entries from $C(X)$ defines a linear operator on $l_{2}(C(X))$ if for every $f=\left\{f_{k}\right\}_{k=1}^{\infty} \in l_{2}(C(X))$, the series $\sum_{k=1}^{\infty} a_{j k} f_{k}$ converges in $C(X)$ for all $j$, and the sequence $\left\{\sum_{k=1}^{\infty} a_{j k} f_{k}\right\}_{j=1}^{\infty}$ belongs to $l_{2}(C(X))$. We denote the sequence $\left\{\sum_{k=1}^{\infty} a_{j k} f_{k}\right\}_{j=1}^{\infty}$ by $A f$ for all $f=\left\{f_{k}\right\}_{k=1}^{\infty} \in l_{2}(C(X))$ and call the operator $f \mapsto A f$ the linear operator defined by $A$. Let $\mathcal{B}\left(l_{2}(C(X))\right)$ be the set of all matrices $A$ over $C(X)$ such that $A$ defines a linear operator on $l_{2}(C(X))$. For any matrix $A=\left[a_{j k}\right]$ over $C(X)$, 
we let, for each $n \in \mathbb{N}, A_{n}$, be the matrix which agrees with $A$ on the upper left $n \times n$ block and is 0 on all other entries. For each $t \in X$, we let $A[t]:=\left[a_{j k}(t)\right]$. For $f=\left\{f_{k}\right\}_{k=1}^{\infty} \in l_{2}(C(X))$, we let, for each $t \in X, f[t]:=\left\{f_{k}(t)\right\}_{k=1}^{\infty}$. It is clear that $f[t] \in l_{2}$ for all $t$ and $\|f\|_{2}=\sup _{t \in X}\|f[t]\|_{2}$.

Proposition $2.1([6])$. If $A \in \mathcal{B}\left(l_{2}(C(X))\right)$, then the linear operator defined by $A$ is bounded.

If $A \in \mathcal{B}\left(l_{2}(C(X))\right)$, we define the norm $\|A\|$ to be the norm of the linear operator defined by $A$.

Proposition 2.2. Let $A$ be a matrix with entries from $C(X)$.

(1) If $A \in \mathcal{B}\left(l_{2}(C(X))\right)$, then $\sup _{t \in X}\|A[t]\|<\infty$. Moreover, $\|A\|=$ $\sup _{t \in X}\|A[t]\|$.

(2) If $A \in \mathcal{B}\left(l_{2}(C(X))\right)$, then $\left\|A_{n\lrcorner}\right\| \nearrow\|A\|$.

Proof. (1). Let $f=\left\{f_{k}\right\}_{k=1}^{\infty} \in l_{2}(C(X))$ with $\|f\|_{2} \leq 1$. Then we get for each $t \in X$ that $\|(A[t]) f[t]\|_{2} \leq\|A[t]\|$. So

$$
\|A\|=\sup \left\{\sup _{t \in X}\|(A[t]) f[t]\|_{2}: f \in l_{2}(C(X)),\|f\|_{2} \leq 1\right\} \leq \sup _{t \in X}\|A[t]\| .
$$

Let $x=\left\{\xi_{k}\right\}_{k=1}^{\infty} \in l_{2}$ with $\|x\|_{2} \leq 1$. For each $k$, we put $f_{k}(t)=\xi_{k}$ for all $t \in X$ and $f_{x}=\left\{f_{k}\right\}_{k=1}^{\infty}$. Then $f_{x} \in l_{2}(C(X))$ and $\left\|f_{x}\right\|_{2}=\|x\|_{2} \leq 1$. Thus, for each $t \in X,\|A[t] x\|_{2}=\left\|A[t] f_{x}[t]\right\|_{2} \leq\|A\|$. This implies that $\|A[t]\| \leq\|A\|$ for all $t$. The proof is complete.

(2). Suppose that $A \in \mathcal{B}\left(l_{2}(C(X))\right)$. Then by the assertion (1) above, $A[t] \in \mathcal{B}\left(l_{2}\right)$ for all $t \in X$. So, we have $A_{n_{\lrcorner}}[t] \nearrow A[t]$ for all $t$. Hence we get by (1) again that $\left\|A_{n\lrcorner}\right\| \leq\left\|A_{n+1}\right\| \leq\|A\|$ for all $n$. This implies that $A_{n} \nearrow \sup _{n}\left\|A_{n_{\lrcorner}}\right\|$and $\sup _{n}\left\|A_{n_{\lrcorner}}\right\| \leq\|A\|$. To see that $\|A\| \leq \sup _{n}\left\|A_{n}\right\|$, let $\epsilon>0$ be given. Then by (1), there exists $s \in X$ such that $\|A\|<\|A[s]\|+\epsilon$. This implies that there is a positive integer $n_{0}$ such that

$$
\|A\|<\left\|A_{\left.n_{0}\right\lrcorner}[s]\right\|+\epsilon \leq\left\|A_{\left.n_{0}\right\lrcorner}\right\|+\epsilon \leq \sup _{n}\left\|A_{n_{\lrcorner}}\right\|+\epsilon .
$$

Since $\epsilon$ is arbitrary, $\|A\| \leq \sup _{n}\left\|A_{n_{\lrcorner}}\right\|$.

The following example shows that for a matrix $A$ over $C(X)$, the finiteness of $\sup _{t \in X}\|A[t]\|$ does not necessarily imply the boundedness of $A$.

Example 2.3. Let $X=[0,1]$ and let $A$ be the matrix whose the first column is the sequence $f\langle 2\rangle$ given in Example 1.1 and all other columns 0. Then $\sup _{t \in X}\|A[t]\| \leq 1$, but $A$ does not define a linear operator on $l_{2}(C([0,1]))$ since $A x=f\langle 2\rangle$, where $x=\{1,0,0,0, \ldots\}$, does not belong to $l_{2}(C([0,1]))$.

Lemma 2.4. If $A$ is a matrix with entries from $C(X)$ and the set $\left\{\left\|A_{n_{\lrcorner}}\right\|\right.$: $n \in \mathbb{N}\}$ is bounded, then $A f$ is a sequence in $C(X)$ for all $f \in l_{2}(C(X))$. 
Proof. Let $M=\sup _{n \in \mathbb{N}}\left\|A_{n_{\lrcorner}}\right\|$and $f=\left\{f_{k}\right\}_{k=1}^{\infty} \in l_{2}(C(X))$. For any $n>$ $m \in \mathbb{N}$, we let $f_{[m, n]}:=\left\{0,0, \ldots, 0, f_{m}, f_{m+1}, \ldots, f_{n}, 0,0, \ldots\right\}$, clearly, $f_{[m, n]} \in$ $l_{2}(C(X))$. Let $j \in \mathbb{N}$ and $\epsilon>0$. Then there exists a positive integer $N$ such that

$$
\left\|\sum_{k=m}^{n}\left|f_{k}\right|^{2}\right\|_{C(X)}<\left(\frac{\epsilon}{M}\right)^{2} \text { for all } n>m>N .
$$

So, if $n>m>\max \{j, N\}$, we get that

$$
\begin{aligned}
\left\|\sum_{k=m}^{n} a_{j k} f_{k}\right\| & =\sup _{t \in X}\left|\sum_{k=m}^{n} a_{j k}(t) f_{k}(t)\right| \leq\left\|A_{n\lrcorner} f_{[m, n]}\right\|_{2} \\
& \leq\left\|A_{n_{\lrcorner}}\right\|\left\|f_{[m, n]}\right\|_{2} \leq M\left\|\sum_{k=m}^{n}\left|f_{k}\right|^{2}\right\|_{C(X)}^{\frac{1}{2}} \\
& <M\left(\frac{\epsilon}{M}\right)=\epsilon .
\end{aligned}
$$

Hence $\left\{\sum_{k=1}^{n} a_{j k} f_{k}\right\}_{n=1}^{\infty}$ is a Cauchy sequence in $C(X)$, so it is convergent.

Remark 2.5. If $X$ is a singleton, then the assumption of the above lemma implies that $A \in \mathcal{B}\left(l_{2}(C(X))\right)$. This is not true in general. Indeed, from Example 2.3, we also have by Proposition 2.2(1) that $\left\|A_{n_{\lrcorner}}\right\| \leq 1$ for all $n$, but $A$ does not belong to $\mathcal{B}\left(l_{2}(C(X))\right)$. The following proposition tells us when the boundedness of the set $\left\{\left\|A_{n}\right\|: n \in \mathbb{N}\right\}$, which is clearly equivalent to the finiteness of $\sup _{t \in X}\|A[t]\|$, implies the boundedness of the matrix $A$.

Proposition 2.6. Suppose that $A$ is a matrix over $C(X)$ with $A[t] \in \mathcal{B}\left(l_{2}\right)$ for all $t \in X$ and the function $t \mapsto A[t]$ from $X$ into $\mathcal{B}\left(l_{2}\right)$ is continuous. Then $A \in \mathcal{B}\left(l_{2}(C(X))\right)$.

Proof. Since the function $t \mapsto A[t]$ is continuous and $X$ is compact, $\sup _{t \in X}\|A[t]\|<\infty$. Let $M=\sup _{t \in X}\|A[t]\|$. Then for each $n,\left\|A_{n_{\lrcorner}}[t]\right\| \leq$ $\|A[t]\| \leq M$ for all $t$. Thus, by the Proposition 2.2(1) and Lemma 2.4, Af is a sequence in $C(X)$ for all $f \in l_{2}(C(X))$. We now want to show that Af $\in l_{2}(C(X))$ for all $f \in l_{2}(C(X))$. Let $f \in l_{2}(C(X))$. Then by Proposition 1.2, the function $t \mapsto f[t]$ from $X$ into $l_{2}$ is continuous. For each $t \in X$, we have $A[t] \in \mathcal{B}\left(l_{2}\right)$. So $A f[t]=A[t] f[t] \in l_{2}$ for all $t$. It follows that the function $t \mapsto A f[t]$ from $X$ into $l_{2}$ is well defined. For any $s, t \in X$, we have

$$
\begin{aligned}
\|A f[s]-A f[t]\|_{2} & =\|A[s] f[s]-A[t] f[t]\|_{2} \\
& \leq\|A[s] f[s]-A[s] f[t]\|_{2}+\|A[s] f[t]-A[t] f[t]\|_{2} \\
& \leq\|A[s]\|\|f[s]-f[t]\|_{2}+\|A[s]-A[t]\|\|f[t]\|_{2} \\
& \leq M\|f[s]-f[t]\|_{2}+\|A[s]-A[t]\|\|f\|_{2} .
\end{aligned}
$$

Hence, by the continuity of the functions $t \mapsto A[t]$ and $t \mapsto f[t]$, we obtain that the function $t \mapsto A f[t]$ is continuous. So, by Proposition 1.2, Af $\in$ $l_{2}(C(X))$. 
Theorem 2.7. $\mathcal{B}\left(l_{2}(C(X))\right)$ equipped with the operator norm is a Banach space. Furthermore, $\mathcal{B}\left(l_{2}(C(X))\right)$ contains the identity operator, and for $A=$ $\left[a_{j i}\right], B=\left[a_{i k}\right] \in \mathcal{B}\left(l_{2}(C(X))\right)$, the matrix

$$
A B:=\left[\sum_{i=1}^{\infty} a_{j i} b_{i k}\right]
$$

belongs to $\mathcal{B}\left(l_{2}(C(X))\right)$ and $(A B) f=A(B f)$ for all $f \in l_{2}(C(X))$. In other words, $\mathcal{B}\left(l_{2}(C(X))\right)$ is a Banach subalgebra with identity of the Banach algebra of all bounded linear operators on $l_{2}(C(X))$.

Proof. Let $\left\{A_{n}=\left[a_{j k}^{(n)}\right]\right\}_{n=1}^{\infty}$ be a Cauchy sequence in $\mathcal{B}\left(l_{2}(C(X))\right)$. By Proposition 2.2(1), we have for each $(j, k) \in \mathbb{N} \times \mathbb{N}$ and $t \in X$ that

(*) $\quad\left|a_{j k}^{(n)}(t)-a_{j k}^{(m)}(t)\right| \leq\left\|A_{n}[t]-A_{m}[t]\right\| \leq\left\|A_{n}-A_{m}\right\|$ for all $n, m$.

Thus, for any $(j, k),\left\|a_{j k}^{(n)}-a_{j k}^{(m)}\right\|_{C(X)} \leq\left\|A_{n}-A_{m}\right\|$ for all $n, m$. So $\left\{a_{j k}^{(n)}\right\}_{n=1}^{\infty}$ is a Cauchy sequence in $C(X)$ for all $(j, k)$. Hence, by completeness of $C(X)$, there exists $a_{j k} \in C(X)$ such that $a_{j k}^{(n)} \rightarrow a_{j k}$ as $n \rightarrow \infty$. Put $A=\left[a_{j k}\right]$. We will show that $A \in \mathcal{B}\left(l_{2}(C(X))\right)$ and $A_{n} \rightarrow A$ as $n \rightarrow \infty$. Let $\nu \in \mathbb{N}$ and $x=\left\{\xi_{k}\right\}_{k=1}^{\infty} \in l_{2}$ with $\|x\|_{2} \leq 1$. Let $M=\sup _{n}\left\|A_{n}\right\|$. Then for each $t \in X$,

$$
\begin{aligned}
\left\|A_{\nu_{\lrcorner}}[t] x\right\|_{2}^{2} & =\sum_{j=1}^{\nu}\left|\sum_{k=1}^{\nu} a_{j k}(t) \xi_{k}\right|^{2} \\
& \leq 4 \sum_{j=1}^{\nu}\left|\sum_{k=1}^{\nu}\left(a_{j k}^{(n)}(t)-a_{j k}(t)\right) \xi_{k}\right|^{2}+4 M^{2} \text { for all } n .
\end{aligned}
$$

By taking the limit as $n \rightarrow \infty$, we get $\left\|A_{\nu_{\lrcorner}}[t] x\right\| \leq 2 M$ for all $t$. Thus $\left\|A_{\nu_{\lrcorner}}[t]\right\| \leq$ $2 M$ for all $t$. It follows from Proposition 2.2(1) that $\left\|A_{\nu_{\lrcorner}}\right\| \leq 2 M$ for all $\nu$. Hence, by Lemma 2.4, $A f$ is a sequence in $C(X)$ for all $f \in l_{2}(C(X))$. Let $\epsilon>0$ be given. Since $\left\{A_{n}\right\}_{n=1}^{\infty}$ is a Cauchy sequence, there exists a positive integer $N$ such that $\left\|A_{n}-A_{m}\right\|<\frac{\epsilon}{2}$ for all $n, m \geq N$. By $(*)$, we also have that $\left\{A_{n}[t]\right\}_{n=1}^{\infty}$ is a Cauchy sequence in $\mathcal{B}\left(l_{2}\right)$ for all $t$. Hence, for each $t$, there exists $B[t]=\left[b_{j k}(t)\right] \in \mathcal{B}\left(l_{2}\right)$ such that $A_{n}[t] \rightarrow B[t]$ as $n \rightarrow \infty$. For each $(j, k)$, we have for every $t$ that $\left|a_{j k}^{(n)}(t)-b_{j k}(t)\right| \leq\left\|A_{n}[t]-B[t]\right\| \rightarrow 0$ as $n \rightarrow \infty$. It follows that $A[t]=B[t]$ for all $t$. Let $f=\left\{f_{k}\right\}_{k=1}^{\infty} \in l_{2}(C(X))$ with $\|f\|_{2} \leq 1$. Then

$$
\begin{aligned}
\sup _{t \in X}\left\|\left(A_{n}[t]-A_{m}[t]\right) f[t]\right\|_{2} & =\left\|\left(A_{n}-A_{m}\right) f\right\|_{2} \leq\left\|A_{n}-A_{m}\right\| \\
& <\frac{\epsilon}{2} \quad \text { for all } n, m \geq N .
\end{aligned}
$$

By taking the limit as $m \rightarrow \infty$, we get

$$
\left\|\left(A_{n}-A\right) f\right\|_{2} \leq \frac{\epsilon}{2} \quad \text { for all } n \geq N
$$


This gives us that $A f \in l_{2}^{b}(C(X))$ and $A_{n} f \rightarrow A f$ in $l_{2}^{b}(C(X))$. Since $A_{n} \in$ $\mathcal{B}\left(l_{2}(C(X))\right)$ for all $n, A_{n} f \in l_{2}(C(X))$ by closedness of $l_{p}(C(X))$ in $l_{p}^{b}(C(X))$. Hence $A f \in l_{2}(C(X))$. Thus $A \in \mathcal{B}\left(l_{2}(C(X))\right)$. Since (**) holds for arbitrary $f \in l_{2}(C(X)),\left\|A_{n}-A\right\|<\epsilon$ for all $n \geq N$. Consequently, $A_{n} \rightarrow A$ as $n \rightarrow \infty$.

It is obvious that the linear operator defined by the matrix with entries in the main diagonal 1 and all other entries 0 is exactly the identity operator on $l_{2}(C(X))$. Let $A=\left[a_{j i}\right], B=\left[b_{i k}\right] \in \mathcal{B}\left(l_{2}(C(X))\right)$. Then for each $k$, $\left\{\sum_{i=1}^{\infty} a_{j i} b_{i k}\right\}_{j=1}^{\infty}=A\left(B e_{k}\right) \in l_{2}(C(X))$, where $e_{k}$ is the sequence with $k$ th coordinate 1 and all other coordinates 0 . Thus the series $\sum_{i=1}^{\infty} a_{j i} b_{i k}$ converges in $C(X)$ for all $(j, k)$. So the matrix $A B=\left[\sum_{i=1}^{\infty} a_{j i} b_{i k}\right]$ is well defined. We will show that $A B$ defines a linear operator on $l_{2}(C(X))$ and $(A B) f=A(B f)$ for all $f \in l_{2}(C(X))$. Let $f=\left\{f_{k}\right\}_{k=1}^{\infty} \in l_{2}(C(X))$. Then we have for every $n$ that

$$
\begin{aligned}
\left\|(A B)_{n\lrcorner} f\right\|_{2} & =\sup _{t \in X}\left(\sum_{j=1}^{n}\left|\sum_{k=1}^{n} \sum_{i=1}^{\infty} a_{j i}(t) b_{i k}(t) f_{k}(t)\right|^{2}\right)^{1 / 2} \\
& =\sup _{t \in X}\left(\sum_{j=1}^{n}\left|\sum_{i=1}^{\infty} \sum_{k=1}^{n} a_{j i}(t) b_{i k}(t) f_{k}(t)\right|^{2}\right)^{1 / 2} \\
& =\left\|A_{n_{\lrcorner}}\left(B f_{n_{\lrcorner}}\right)\right\|_{2} \leq\left\|A_{n_{\lrcorner}}\right\|\|B\|\left\|f_{n_{\lrcorner}}\right\|_{2} \leq\|A\|\|B\|\|f\|_{2} .
\end{aligned}
$$

It follows that $\left\|(A B)_{n_{\lrcorner}}\right\| \leq\|A\|\|B\|$ for all $n$. Hence, by Lemma 2.4, we obtain that $(A B) f$ is a sequence in $C(X)$. Since $A[t]$ and $B[t]$ belong to $\mathcal{B}\left(l_{2}\right)$ for all $t,(A B)[t] f[t]=A[t](B[t] f[t])$. This implies that $(A B) f=A(B(f))$, so $(A B) f \in l_{2}(C(X))$. The proof is complete.

For $A \in \mathcal{B}\left(l_{2}(C(X))\right)$, we have by Proposition $2.2(1)$ that $A[t] \in \mathcal{B}\left(l_{2}\right)$ for all $t \in X$. So the function $c_{A}: X \rightarrow \mathcal{B}\left(l_{2}\right)$ defined by $c_{A}(t)=A[t]$ for all $t \in X$ is well defined. Let $\mathcal{B}_{c}\left(l_{2}(C(X))\right)$ be the set of matrices $A$ in $\mathcal{B}\left(l_{2}(C(X))\right)$ such that the function $c_{A}$ is continuous. For the case where $X$ is a singleton, we have that $\mathcal{B}_{c}\left(l_{2}(C(X))\right)=\mathcal{B}\left(l_{2}(C(X))\right)=\mathcal{B}\left(l_{2}\right)$.

Proposition 2.8. The inclusion $\mathcal{B}_{c}\left(l_{2}(C(X))\right) \subseteq \mathcal{B}\left(l_{2}(C(X))\right)$ can be proper.

Proof. Let $X=[0,1]$ and $A$ be the matrix with the first row the sequence $f\langle 2\rangle=\left\{f_{k}\right\}_{k=1}^{\infty}$ defined in Example 1.1 and all other rows 0. Since $f\langle 2\rangle \in$ $l_{2}^{b}(C(X))$, by Theorem 1.3, we get that $A \in \mathcal{B}\left(l_{2}(C(X))\right)$. Let $t_{n}=1-\frac{1}{n}$ for $n=1,2,3, \ldots$. We have that $t_{n} \rightarrow 1$ as $n \rightarrow \infty$ and $\sum_{k=n}^{2 n}\left|f_{k}\left(t_{n}\right)\right|^{2}=$ $\left(1-\frac{1}{n}\right)^{n}-\left(1-\frac{1}{n}\right)^{2 n+1} \rightarrow \frac{1}{e}-\frac{1}{e^{2}}$ as $n \rightarrow \infty$. Obviously, $A[1]=0$. We claim that $A\left[t_{n}\right]$ does not converge to $A[1]$. Suppose that $A\left[t_{n}\right] \rightarrow A[1]$ as $n \rightarrow \infty$. Fix $0<\epsilon<\frac{1}{e}-\frac{1}{e^{2}}$, then there exists a positive integer $N$ such that $\sum_{k=n}^{2 n}\left|f_{k}\left(t_{n}\right)\right|^{2} \leq\left\|f\langle 2\rangle\left[t_{n}\right]\right\|_{2}^{2}=\left\|A\left[t_{n}\right]\right\|^{2}<\epsilon$ for all $n \geq N$. By letting $n \rightarrow \infty$, we obtain that $\frac{1}{e}-\frac{1}{e^{2}} \leq \epsilon$, which is a contradiction.

Theorem 2.9. $\mathcal{B}_{c}\left(l_{2}(C(X))\right)$ is a Banach subalgebra with identity of $\mathcal{B}\left(l_{2}(C(X))\right)$. 
Proof. To see that $\mathcal{B}_{c}\left(l_{2}(C(X))\right)$ is a Banach space, we will show that it is a closed subspace of $\mathcal{B}\left(l_{2}(C(X))\right)$. Let $\left\{A_{n}\right\}_{n=1}^{\infty}$ be a sequence in $\mathcal{B}_{c}\left(l_{2}(C(X))\right)$ and $A \in \mathcal{B}\left(l_{2}(C(X))\right)$. Suppose that $A_{n} \rightarrow A$ as $n \rightarrow \infty$. We want to show that $A \in \mathcal{B}_{c}\left(l_{2}(C(X))\right)$. Let $\left\{t_{\alpha}\right\}$ be a net in $X$ and suppose that $t_{\alpha} \rightarrow t$ for some $t \in X$. Let $\epsilon>0$ be given. Then there exists a positive integer $N$ such that $\left\|A_{N}-A\right\|<\frac{\epsilon}{3}$. Since $A_{N} \in \mathcal{B}_{c}\left(l_{2}(C(X))\right), A_{N}\left[t_{\alpha}\right] \rightarrow A_{N}[t]$. Hence there exists $\gamma$ such that $\left\|A_{N}\left[t_{\alpha}\right]-A_{N}[t]\right\|<\frac{\epsilon}{3}$ for all $\alpha \succeq \gamma$. So, for $\alpha \succeq \gamma$,

$$
\begin{aligned}
\left\|A\left[t_{\alpha}\right]-A[t]\right\| & \leq\left\|A_{N}\left[t_{\alpha}\right]-A\left[t_{\alpha}\right]\right\|+\left\|A_{N}[t]-A[t]\right\|+\left\|A_{N}\left[t_{\alpha}\right]-A_{N}[t]\right\| \\
& <\frac{\epsilon}{3}+\frac{\epsilon}{3}+\frac{\epsilon}{3}=\epsilon .
\end{aligned}
$$

It follows that $A \in \mathcal{B}_{c}\left(l_{2}(C(X))\right)$. Therefore $\mathcal{B}_{c}\left(l_{2}(C(X))\right)$ is a Banach subspace of $\mathcal{B}\left(l_{2}(C(X))\right)$. It is clear that the identity matrix belongs to $\mathcal{B}_{c}\left(l_{2}(C(X))\right)$. For $A, B \in \mathcal{B}_{c}\left(l_{2}(C(X))\right)$, we have for all $t \in X$ that $\|A[t]\| \leq\|A\|,\|B[t]\| \leq$ $\|B\|$ and $c_{A B}(t)=A B[t]=A[t] B[t]=c_{A}(t) c_{B}(t)$. Since $\mathcal{B}\left(l_{2}\right)$ is a Banach algebra under the composition operation, $c_{A B}$ is continuous.

The following are consequences of Proposition 2.6.

Proposition 2.10. $\mathcal{B}_{c}\left(l_{2}(C(X))\right)$ is equal to the set of all matrices $A$ over $C(X)$ such that $A[t] \in \mathcal{B}\left(l_{2}\right)$ for all $t \in X$ and the function $t \mapsto A[t]$ from $X$ into $\mathcal{B}\left(l_{2}\right)$ is continuous.

Proof. It follows directly from Proposition 2.6.

For $A=\left[a_{j k}\right] \in \mathcal{B}\left(l_{2}(C(X))\right)$, we let $A^{*}=\left[c_{j k}\right]$, where $c_{j k}=\overline{a_{k j}}$ for all $j, k$. In the case where $X$ is a singleton, we have that $A^{*}$ is exactly the adjoint of $A$, so it belongs to $\mathcal{B}\left(l_{2}(C(X))\right)$. In general, this is not true: for example, consider the matrix $A$ with the first row the sequence $f\langle 2\rangle$ given in Example 1.1 and all other rows 0. We have seen from Proposition 2.8 and Example 2.3 that $A \in \mathcal{B}\left(l_{2}(C(X))\right)$ and $A^{*} \notin \mathcal{B}\left(l_{2}(C(X))\right)$.

Proposition 2.11. If $A \in \mathcal{B}_{c}\left(l_{2}(C(X))\right)$, then $A^{*} \in \mathcal{B}_{c}\left(l_{2}(C(X))\right)$.

Proof. It follows immediately from the continuity of the function $B \mapsto B^{*}$ on $\mathcal{B}\left(l_{2}\right)$ and Proposition 2.6.

Corollary 2.12. $\mathcal{B}_{c}\left(l_{2}(C(X))\right)$ equipped with the involution $A \mapsto A^{*}$ is a $C^{*}$ algebra with identity.

Proposition 2.13. $\mathcal{B}_{c}\left(l_{2}(C(X))\right)$ is a Banach algebra (without identity) under the Schur product.

Proof. Let $A, B \in \mathcal{B}_{c}\left(l_{2}(C(X))\right)$. Then by Schur-Bennett's theorem $[1,9]$ : $\mathcal{B}\left(l_{2}\right)$ is a Banach algebra under the Schur product, we obtain that the function $c_{A \bullet B}$ is well defined. Since the functions $c_{A}$ and $c_{B}$ are continuous, and we have $\|A[t]\| \leq\|A\|$ and $\|B[t]\| \leq\|B\|$ for all $t$, the function $c_{A} \bullet B$ is continuous. Thus, by Proposition 2.6, $A \bullet B \in \mathcal{B}_{c}\left(l_{2}(C(X))\right)$. By Schur-Bennett's theorem again, we obtain $\|(A \bullet B)[t]\|=\|A[t] \bullet B[t]\| \leq\|A[t]\|\|B[t]\| \leq\|A\|\|B\|$ for all $t$. It 
follows from Proposition 2.2(1) that $\|A \bullet B\| \leq\|A\|\|B\|$. So $\mathcal{B}_{c}\left(l_{2}(C(X))\right)$ is a Banach algebra under the Schur product.

We do not however know if $\mathcal{B}\left(l_{2}(C(X))\right)$ is closed under the Schur product.

\section{Schatten classes of matrices in $\mathcal{B}_{c}\left(l_{2}(C(X))\right)$}

Let $\mathcal{M}_{0}$ be the set of matrices over $C(X)$ having finitely many nonzero entries, and $\mathcal{K}(C(X))$ be the closure of $\mathcal{M}_{0}$ in $\mathcal{B}\left(l_{2}(C(X))\right)$.

Proposition 3.1. $\mathcal{K}(C(X)) \varsubsetneqq \mathcal{B}_{c}\left(l_{2}(C(X))\right)$.

Proof. It is easy to see that $\mathcal{M}_{0} \subseteq \mathcal{B}_{c}\left(l_{2}(C(X))\right)$. Hence, by Theorem 2.9, $\mathcal{K}(C(X)) \subseteq \mathcal{B}_{c}\left(l_{2}(C(X))\right)$. Since the identity matrix does not belong to $\mathcal{K}(C(X))$, the inclusion is proper.

Proposition 3.2. $A \in \mathcal{K}(C(X))$ if and only if $\left\|A_{n_{\lrcorner}}-A\right\| \rightarrow 0$ as $n \rightarrow \infty$.

Proof. Suppose that $A \in \mathcal{K}(C(X))$ and let $\epsilon>0$. Then there exists $B \in \mathcal{M}_{0}$ such that $\|A-B\|<\frac{\epsilon}{2}$. Let $N$ be a positive integer such that $B_{N_{\lrcorner}}=B$. Then for $n \geq N, A_{n\lrcorner}-B=(A-B)_{n\lrcorner}$. Hence, by Proposition 2.2(2), we have

$$
\begin{aligned}
\left\|A_{n\lrcorner}-A\right\| & \leq\|A-B\|+\left\|A_{n_{\lrcorner}}-B\right\|=\|A-B\|+\left\|(A-B)_{n_{\lrcorner}}\right\| \\
& \leq 2\|A-B\|<\epsilon \text { for all } n \geq N .
\end{aligned}
$$

The converse is obvious.

Let $\mathcal{K}$ be the class of compact operators on $l_{2}$. If $X$ is a singleton, then $\mathcal{K}(C(X))=\mathcal{K}$.

Proposition 3.3. $\mathcal{K}(C(X))=\left\{A \in \mathcal{B}_{c}\left(l_{2}(C(X))\right): A[t] \in \mathcal{K}\right.$ for all $\left.t \in X\right\}$.

Proof. Suppose that $A \in \mathcal{B}_{c}\left(l_{2}(C(X))\right)$ with $A[t] \in \mathcal{K}$ for all $t \in X$. Let $\epsilon>0$ be given. Then by the continuity of the function $c_{A}$, we get for each $t \in X$ that there exists an open set $U(t)$ in $X$ such that $t \in U(t)$ and

$$
\|A[t]-A[s]\|<\frac{\epsilon}{4} \text { for all } s \in U(t) .
$$

Since $X$ is compact, there exist $t_{1}, t_{2}, \ldots, t_{m} \in X$ such that $X=U\left(t_{1}\right) \cup U\left(t_{2}\right) \cup$ $\cdots \cup U\left(t_{m}\right)$. Since $A\left[t_{i}\right] \in \mathcal{K}$ for all $i \in\{1,2, \ldots, m\}$, there exists, for each $i$, a positive integer $N_{i}$ such that

$$
\left\|A_{n_{\lrcorner}}\left[t_{i}\right]-A\left[t_{i}\right]\right\|<\frac{\epsilon}{4} \text { for all } n \geq N_{i} .
$$

Put $N=\max \left\{N_{1}, N_{2}, \ldots, N_{m}\right\}$ and let $s \in X$. Then there exists $i_{0} \in$ $\{1,2, \ldots, m\}$ such that $s \in U\left(t_{i_{0}}\right)$. Hence if $n \geq N$, we have that

$$
\begin{aligned}
\left\|A_{n_{\lrcorner}}[s]-A[s]\right\| & \leq\left\|A_{n_{\lrcorner}}\left[t_{i_{0}}\right]-A_{n_{\lrcorner}}[s]\right\|+\left\|A_{n_{\lrcorner}}\left[t_{i_{0}}\right]-A\left[t_{i_{0}}\right]\right\|+\left\|A\left[t_{i_{0}}\right]-A[s]\right\| \\
& \leq 2\left\|A\left[t_{i_{0}}\right]-A[s]\right\|+\left\|A_{n_{\lrcorner}}\left[t_{i_{0}}\right]-A\left[t_{i_{0}}\right]\right\|<\frac{2 \epsilon}{4}+\frac{\epsilon}{4}=\frac{3 \epsilon}{4} .
\end{aligned}
$$

Thus, by Proposition 2.2(1), we obtain that

$$
\left\|A_{n\lrcorner}-A\right\|<\epsilon \text { for all } n \geq N \text {. }
$$


So $A \in \mathcal{K}(C(X))$. The reverse inclusion follows immediately from Proposition 3.1 and Proposition 2.2(1). The proof is complete.

Corollary 3.4. $\mathcal{K}(C(X))$ is a proper closed ideal of $\mathcal{B}_{c}\left(l_{2}(C(X))\right)$.

Proof. If $A \in \mathcal{K}(C(X))$ and $B \in \mathcal{B}_{c}\left(l_{2}(C(X))\right)$, then $A B[t]$ and $B A[t]$ are elements of $\mathcal{K}$ for all $t$. Hence, by proposition above, both $A B$ and $B A$ belong to $\mathcal{K}(C(X))$. Consequently, $\mathcal{K}(C(X))$ is an ideal of $\mathcal{B}_{c}\left(l_{2}(C(X))\right)$.

The following example shows us that $\mathcal{K}(C(X))$ may not be an ideal of $\mathcal{B}\left(l_{2}(C(X))\right)$.

Example 3.5. Let $X=[0,1]$, let $A$ be the matrix whose $(1,1)$ entry is 1 and all other entries 0 , and let $B$ be the matrix with the first row the sequence $f\langle 2\rangle$ given in Example 1.1 and all other rows 0. Clearly, $A B=B$. We have seen from Proposition 2.8 that $B \in \mathcal{B}\left(l_{2}(C([0,1]))\right) \backslash \mathcal{B}_{c}\left(l_{2}(C([0,1]))\right)$.

If $A \in \mathcal{K}(C(X))$, then $A[t] \in \mathcal{K}$ for all $t \in X$. For each $t \in X$, let $\left\{s_{n}(A[t])\right\}_{n=1}^{\infty}$ be the sequence of singular values of $A[t]$. For each $n \in \mathbb{N}$, let $\widetilde{s}_{n}(A): X \rightarrow[0, \infty)$ be the function defined by

$$
\widetilde{s}_{n}(A)(t)=s_{n}(A[t]) \text { for all } t \in X .
$$

Theorem 3.6. For $A \in \mathcal{K}(C(X))$, the function $\widetilde{s}_{n}(A)$ is continuous for all $n$. Furthermore, $\widetilde{s}_{1}(A)(t) \geq \widetilde{s}_{2}(A)(t) \geq \cdots \geq 0$ for all $t \in X$ and $\widetilde{s}_{n}(A) \rightarrow 0$ as $n \rightarrow \infty$ in $C(X)$.

Proof. Let $A \in \mathcal{K}(C(X))$. For each $n \in \mathbb{N}$, we defined a function $f_{n}: \mathcal{K} \rightarrow$ $[0, \infty)$ by $f_{n}(B)=s_{n}(B)$ for all $B \in \mathcal{K}$. Clearly, $\widetilde{s}_{n}(A)=f_{n} c_{A}$ for all $n$, hence, by Proposition 3.1, the continuity of $\widetilde{s}_{n}(A)$ will be proved once we can show that $f_{n}$ is continuous. The continuity of $f_{n}$ follows directly from the fact that $\left|s_{n}(B)-s_{n}(C)\right| \leq\|B-C\|$ for all $B, C \in \mathcal{K}$. It is clear that for every $t \in X, \widetilde{s}_{1}(A)(t) \geq \widetilde{s}_{2}(A)(t) \geq \cdots \geq 0$. Since $X$ is compact and $\widetilde{s}_{n}(A)(t) \rightarrow 0$ as $n \rightarrow \infty$ for all $t \in X,\left\|\widetilde{s}_{n}(A)\right\|_{C(X)} \rightarrow 0$ as $n \rightarrow \infty$.

For $1 \leq p<\infty$, we define three classes of matrices in $\mathcal{K}(C(X))$ as follows:

$$
\begin{aligned}
\mathcal{C}_{p}^{b}(C(X))=\left\{A \in \mathcal{K}(C(X)):\left\{\widetilde{s}_{n}(A)\right\}_{n=1}^{\infty} \in l_{p}^{b}(C(X))\right\} \\
\mathcal{C}_{p}(C(X))=\left\{A \in \mathcal{K}(C(X)):\left\{\widetilde{s}_{n}(A)\right\}_{n=1}^{\infty} \in l_{p}(C(X))\right\} \\
\mathcal{C}_{p}^{c}(C(X))=\left\{A \in \mathcal{K}(C(X)): \text { the function } t \mapsto A[t] \text { from } X \text { into } \mathcal{C}_{p}\right. \\
\text { is continuous }\} .
\end{aligned}
$$

It is clear that $A \in \mathcal{C}_{p}^{b}(C(X))$ if and only if $A \in \mathcal{K}(C(X))$ and $\sup _{t \in X} \mid\|A[t]\| \|_{p}<$ $\infty$.

Proposition 3.7. $\mathcal{C}_{p}^{c}(C(X)) \subseteq \mathcal{C}_{p}(C(X)) \subseteq \mathcal{C}_{p}^{b}(C(X))$. 
Proof. It is obvious that both $\mathcal{C}_{p}^{c}(C(X))$ and $\mathcal{C}_{p}(C(X))$ are subsets of $\mathcal{C}_{p}^{b}(C(X))$. We will show that $\mathcal{C}_{p}^{c}(C(X)) \subseteq \mathcal{C}_{p}(C(X))$. Suppose that $A \in \mathcal{C}_{p}^{c}(C(X))$. Then the function $t \mapsto A[t]$ from $X$ into $\mathcal{C}_{p}$ is continuous. This implies that the function $t \mapsto \mid\|A[t]\|\left\|_{p}=\right\|\left\{\widetilde{s}_{k}(A)(t)\right\}_{k=1}^{\infty} \|_{p}$ is continuous. It follows from Proposition 1.2 that $\left\{\widetilde{s}_{k}(A)\right\}_{k=1}^{\infty} \in l_{p}(C(X))$, so $A \in \mathcal{C}_{p}(C(X))$.

The following example shows that the inclusion $\mathcal{C}_{p}(C(X)) \subseteq \mathcal{C}_{p}^{b}(C(X))$ can be proper. So the inclusion $\mathcal{C}_{p}^{c}(C(X)) \subseteq \mathcal{C}_{p}^{b}(C(X))$ can also be proper. For the space $l_{p}(C(X))$, we have that $f \in l_{p}(C(X))$ if and only if the function $t \mapsto f[t]$ from $X$ into $l_{p}$ is continuous. We expect to have a similar characterization for $\mathcal{C}_{p}(C(X))$, i.e., $A \in \mathcal{C}_{p}(C(X))$ if and only if the function $t \mapsto A[t]$ from $X$ into $\mathcal{C}_{p}$ is continuous (or equivalently, $\mathcal{C}_{p}(C(X))=\mathcal{C}_{p}^{c}(C(X))$ ). Now, we have $\mathcal{C}_{p}^{c}(C(X)) \subseteq \mathcal{C}_{p}(C(X))$, but we do not know if we have equality or an example of proper inclusion.

Example 3.8. $\mathcal{C}_{p}(C(X)) \varsubsetneqq \mathcal{C}_{p}^{b}(C(X))$. Let $X=[0,1]$ and let $A$ be the matrix with the main diagonal the sequence $f\langle p\rangle=\left\{f_{k}\right\}_{k=1}^{\infty}$ given in Example 1.1 and all other entries 0 . It is easy to see that $A \in \mathcal{B}\left(l_{2}(C([0,1]))\right)$. Since $f_{k} \rightarrow 0$ as $n \rightarrow \infty$ in $C(X), A \in \mathcal{K}(C([0,1]))$. It is clear that $\widetilde{s}_{k}(A)=f_{k}$ for all $k$. Hence $A \in \mathcal{C}_{p}^{b}(C([0,1])) \backslash \mathcal{C}_{p}(C([0,1]))$.

It is easy to see that $\mathcal{C}_{p}^{b}(C(X))$ and $\mathcal{C}_{p}^{c}(C(X))$ are linear spaces. For the case where $X$ is infinite, we do not know if $\mathcal{C}_{p}(C(X))$ is closed under addition.

Theorem 3.9. $\mathcal{C}_{p}^{b}(C(X))$ and $\mathcal{C}_{p}^{c}(C(X))$ equipped with the norm

$$
\|A\|\left\|_{p}:=\sup _{t \in X} \mid\right\| A[t] \|_{p}
$$

are Banach spaces.

Proof. Let $\left\{A_{n}\right\}_{n=1}^{\infty}$ be a Cauchy sequence in $\mathcal{C}_{p}^{b}(C(X))$. Note that for any $B \in \mathcal{C}_{p},\|B\|=s_{1}(B) \leq\|B\|_{p}$. This gives us that $\left\{A_{n}\right\}_{n=1}^{\infty}$ is also a Cauchy sequence in $\mathcal{K}(C(X))$. So there exists $A$ in $\mathcal{K}(C(X))$ such that $A_{n} \rightarrow A$ in $\mathcal{K}(C(X))$. We will show that $A \in \mathcal{C}_{p}^{b}(C(X))$ and $A_{n} \rightarrow A$ as $n \rightarrow \infty$. Since $\left\{A_{n}\right\}_{n=1}^{\infty}$ is a Cauchy sequence in $\mathcal{C}_{p}^{b}(C(X)),\left\{A_{n}[t]\right\}_{n=1}^{\infty}$ is a Cauchy sequence in $\mathcal{C}_{p}$ for all $t \in X$. Thus, for each $t$, we have by completeness of $\mathcal{C}_{p}$ that there exists $A_{t}$ in $\mathcal{C}_{p}$ such that $A_{n}[t] \rightarrow A_{t}$ as $n \rightarrow \infty$. From this, we have $A_{n}[t] \rightarrow A_{t}$ in $\mathcal{K}$ for all $t$. Since $A_{n} \rightarrow A$ in $\mathcal{K}(C(X)), A_{n}[t] \rightarrow A[t]$ in $\mathcal{K}$ for all $t$. Hence $A[t]=A_{t}$ for all $t$. Let $\epsilon>0$ be given. Then there exists a positive integer $N$ such that for any $t,\|\| A_{n}[t]-A_{m}[t]\|\|_{p} \leq\left\|A_{n}-A_{m}\right\| \|_{p} \leq \frac{\epsilon}{2}$ for all $n, m \geq N$. By taking the limit as $m \rightarrow \infty$, we obtain for each $t$ that \|\|$A_{n}[t]-A[t] \|_{p} \leq \frac{\epsilon}{2}$ for all $n \geq N$. It follows that $\left\|A_{n}-A\right\|\left\|_{p}=\sup _{t \in X}\right\| A_{n}[t]-A[t]\|\|_{p} \leq \frac{\epsilon}{2}<\epsilon$ for all $n \geq N$. This gives us that $A \in \mathcal{C}_{p}^{b}(C(X))$ and $A_{n} \rightarrow A$ as $n \rightarrow \infty$. Accordingly, $\mathcal{C}_{p}^{b}(C(X))$ is a Banach space.

To see that $\mathcal{C}_{p}^{c}(C(X))$ is a Banach space, we will show that it is a closed subspace of $\mathcal{C}_{p}^{b}(C(X))$. Let $\left\{A_{n}\right\}_{n=1}^{\infty}$ be a sequence in $\mathcal{C}_{p}^{c}(C(X))$ and $A \in$ 
$\mathcal{C}_{p}^{b}(C(X))$. Suppose that $A_{n} \rightarrow A$ as $n \rightarrow \infty$. Let $\left\{t_{\alpha}\right\}$ be a net in $X$ such that $t_{\alpha} \rightarrow t$ for some $t \in X$. We want to show that $\left\|A\left[t_{\alpha}\right]-A[t]\right\| \rightarrow 0$. Let $\epsilon>0$. Then there exists a positive integer $N$ such that $\left\|A_{N}-A\right\|_{p}<\frac{\epsilon}{3}$. Since $A_{N} \in \mathcal{C}_{p}^{c}(C(X))$, there is $\gamma$ such that $\left\|A_{N}\left[t_{\alpha}\right]-A_{N}[t]\right\|_{p}<\frac{\epsilon}{3}$ for all $\alpha \succeq \gamma$. So $\left\|A\left[t_{\alpha}\right]-A[t]\right\|\left\|_{p} \leq\right\| A_{N}\left[t_{\alpha}\right]-A\left[t_{\alpha}\right]\|\|_{p}+\|\| A_{N}[t]-A[t]\|\|_{p}+\|\| A_{N}\left[t_{\alpha}\right]-A_{N}[t]\|\|_{p}$ $<\frac{\epsilon}{3}+\frac{\epsilon}{3}+\frac{\epsilon}{3}=\epsilon$ for all $\alpha \succeq \gamma$.

The proof is complete.

Proposition 3.10. $\mathcal{C}_{p}^{b}(C(X))$ and $\mathcal{C}_{p}^{c}(C(X))$ are ideals of $\mathcal{B}_{c}\left(l_{2}(C(X))\right)$.

Proof. We will first show that $\mathcal{C}_{p}^{b}(C(X))$ is an ideal of $\mathcal{B}_{c}\left(l_{2}(C(X))\right)$. Let $A \in \mathcal{C}_{p}^{b}(C(X))$ and $B \in \mathcal{B}_{c}\left(l_{2}(C(X))\right)$. Then by Corollary 3.4 , both $A B$ and $B A$ belong to $\mathcal{K}(C(X))$. Since $A[t] \in \mathcal{C}_{p}$ and $B[t] \in \mathcal{B}\left(l_{2}\right)$ for all $t \in X$, $\|(A B)[t]\|\left\|_{p} \leq\right\| A[t]\|\|_{p}\|B[t]\| \leq\|\| A\|\|_{p}\|B\|$. Similarly, we have $\|(B A)[t]\| \|_{p} \leq$ \|\|$A\left\|_{p}\right\| B \|$ for all $t$. This implies that both $A B$ and $B A$ belong to $\mathcal{C}_{p}^{b}(C(X))$, so $\mathcal{C}_{p}^{b}(C(X))$ is an ideal of $\mathcal{B}_{c}\left(l_{2}(C(X))\right)$.

To show that $\mathcal{C}_{p}^{c}(C(X))$ is an ideal of $\mathcal{B}_{c}\left(l_{2}(C(X))\right)$, suppose that $A \in$ $\mathcal{C}_{p}^{c}(C(X))$ and $B \in \mathcal{B}_{c}\left(l_{2}(C(X))\right)$. We will show that $A B \in \mathcal{C}_{p}^{c}(C(X))$. By the fact above, we have $A B \in \mathcal{C}_{p}^{b}(C(X))$. For any $s, t \in X$, we have

$$
\begin{aligned}
\|A B[s]-A B[t]\| \|_{p} & =\left.\|A[s] B[s]-A[t] B[t]\|\right|_{p} \\
& \leq\|A[s] B[s]-A[s] B[t]\|\left\|_{p}+\right\| A[s] B[t]-A[t] B[t]\|\|_{p} \\
& \leq\|A[s]\|\left\|_{p}\right\| B[s]-B[t]\|+\| A[s]-A[t]\left\|_{p}\right\| B[t] \| \\
& \leq\|A\|\left\|_{p}\right\| B[s]-B[t]\|+\| A[s]-A[t]\left\|_{p}\right\| B \| .
\end{aligned}
$$

So, by the assumption, the function $t \mapsto A B[t]$ is continuous. This means that $A B \in \mathcal{C}_{p}^{c}(C(X))$. By using a similar argument, we also have that $B A \in$ $\mathcal{C}_{p}^{c}(C(X))$. It follows that $\mathcal{C}_{p}^{c}(C(X))$ is an ideal of $\mathcal{B}_{c}\left(l_{2}(C(X))\right)$.

\section{References}

[1] G. Bennett, Schur multipliers, Duke Math. J. 44 (1977), no. 3, 603-639.

[2] K. R. Davidson, Nest Algebras, Longman Scientific and Technical, Essex, UK, 1988.

[3] J. Dixmier, Les fonctionnelles lin'earies sur l'ensemble des opérateurs bornés d'un espace de Hilbert, Ann. of Math. (2) 51 (1950). 387-408.

[4] K. Fan, Maximum properties and inequalities for the eigenvalues of completely continuous operators, Proc. Nat. Acad. Sci., U. S. A. 37 (1951), 760-766.

[5] I. C. Gohberg and M. G. Kreìn, Introduction to the Theory of Linear Nonselfadjoint Operators, "Nauka", Moscow, 1965; English transl., Transl. Math. Monograph, 18, Amer. Math. Soc, Providence, RI, 1969.

[6] L. Livshits, S.-C. Ong, and S.-W. Wang, Schur algebras over function algebras, Houston J. Math. 30 (2004), no. 4, 1195-1217.

[7] J. Rakbud, O. Wootijiruttikal, and P. Chaisuriya, Spaces of sequences over function algebras, J. Anal. Appl. 5 (2007), no. 3, 185-199.

[8] R. Schatten, A Theory of Cross-Spaces, Annals of Mathematics Studies, no. 26. Princeton University Press, Princeton, N. J., 1950. 
[9] J. Schur, Bemerkungen Theorie der beschranken Bilineärformen mit unendlich vielen Veränder lichen, J. Reine Angew. Math., 1911.

JITTI RAKBUD

Department of Mathematics

FACULTy OF SCIENCE

SILPAKORN UNIVERSITY

NaKorn Pathom 73000, Thailand

E-mail address: jitti@su.ac.th

Pachara Chaisuriya

Department of Mathematics

FACUlTy OF SCIENCE

MAHIDOL UNIVERSITY

BANGKOK 10400, THAILAND

E-mail address: scpcs@mahidol.ac.th 